\title{
Complete Long-Term Remission of an Inflammatory Pseudotumor under Corticosteroid Therapy
}

\author{
Lukas Pfeifer $^{a} \quad$ Abbas Agaimy $^{c}$ Rolf Jankab \\ Frank Boxberger $^{\mathrm{a}} \quad$ Axel Wein $^{\mathrm{a}} \quad$ Markus F. Neurath $^{\mathrm{a}}$ \\ Jürgen Siebler ${ }^{\mathrm{a}}$
}

Departments of anternal Medicine 1 and ${ }^{b}$ Radiology, and Institute of Pathology, University of Erlangen, Erlangen, Germany

\section{Key Words}

Inflammatory pseudotumor - Skeletal back muscle · Fracture · Corticosteroid · Complete remission

\begin{abstract}
Inflammatory pseudotumors (IPT) form a group of etiologically, histologically, and biologically heterogeneous tumefactive lesions that are histologically characterized by prominent inflammatory infiltrates. IPT has been described in various organs including the lungs, bladder, liver, spleen, heart, and others. It may mimic a malignant tumor clinically and radiologically. We report a case of a 26-year-old woman with an ALK1negative IPT (7 cm in maximal diameter) mainly located in the 12 th right back muscles, surrounding a fractured rib. Histologically, the tumor consisted of an inflammatory infiltrate composed predominantly of diffusely distributed lymphoplasmacytic cells and stromal fibroblasts associated with focal obliterative phlebitis. Conservative steroid treatment resulted in complete remission and the patient remained disease-free for more than 1 year later. To our knowledge this is the first report of IPT involving the skeletal back muscle and complete resolution under corticosteroid treatment.
\end{abstract}

\section{Introduction}

The term 'inflammatory pseudotumor' (IPT) has historically been applied to a heterogeneous group of mass-forming lesions characterized by a prominent inflammatory infiltrate as the predominant cellular component. However, several lesions previously considered variants of IPT have been identified as specific entities; some of them 
represent true neoplasms. The neoplastic variants of IPT include inflammatory myofibroblastic tumor (IMT) associated with ALK-1 translocation [1] and IPT-like follicular dendritic cell tumor of the liver and spleen related to clonal Epstein-Barr virus (EBV) infection [2]. In contrast, mycobacterial spindle-cell IPT of lymph node [3] and tumefactive lesions associated with immunoglobulin G4 (IgG4) [4] represent examples of infectious and autoimmune-induced IPT, respectively. Orbital IPT was identified recently as a member of IgG4-related IPT [5]. The etiology and pathogenesis of IPT of lymph node and a variety of tumorous inflammatory lesions lacking features of the entities listed above (IPT, not otherwise specified) remain unclear; both reactive-infectious and immunological/idiopathic factors might play a role in their initiation and growth. Given the controversy that accompanied the natural history of IPT and the different criteria used by different authorities in their diagnosis, it is difficult to obtain clear evidence about the incidence, anatomical distribution and malignant characteristics of IPTs in the literature. For example IMTs, which were formerly regarded as IPTs, are now classified as neoplastic lesions. Coffin et al. [6] reported 59 cases of IMT of which 6 had metastasized. In contrast, the entities IPT of lymph nodes and spleen are referred to as benign lesions [3].

We report a case of an IPT consisting of an inflammatory infiltrate composed predominantly of diffusely distributed plasma cells and lymphoid cells, involving the skeletal paravertebral muscle of the back and associated with a fractured rib with complete remission under corticosteroid treatment.

\section{Case Report}

A 26-year-old woman presented with an 8-month history of right-sided back pain of an intermediate severity. She also noticed a growing mass at the right back. The pain started suddenly in the morning with a sound 'like a breaking bone' without trauma. However, the patient reported a history of trauma half a year before the onset of symptoms, when somebody crashed into her right back on a swimming pool slide. After that trauma she had had only some mild pain for a couple of days. The patient also suffered from depression since her teens which required intermediate admissions. She was treated with paroxetine, a selective serotonin reuptake inhibitor, and opipramol, a tricyclic antidepressant, but took no other medication. The patient was married, and a housewife with two male children. She did use tobacco but no alcohol or drugs. Her grandfather had died of pancreatic cancer at the age of 60 , and her uncle had died of a brain tumor at the age of 52. There was no other history of tumors in the family.

At presentation, the patient had a normal weight of $67 \mathrm{~kg}$ and a height of $157 \mathrm{~cm}$. Her blood pressure was at the lower normal level with $105 / 60 \mathrm{~mm} \mathrm{Hg}$ and a pulse of 96 beats per minute. On physical examination there was a painful mass at the right back. It could not be mobilized and was extremely painful so that the patient had severe problems sleeping, especially on the right side. Laboratory results showed an increased C-reactive protein $(41 \mathrm{mg} / \mathrm{l})$ with a normal complete blood count, serum electrolytes and creatinine.

With a clinical suspicion of a sarcoma, a computed tomography (CT) scan with i.v. contrast (88 ml of Imeron 400; Bracco, Konstanz, Germany) was performed which revealed a tumor measuring $7.3 \times 7.1$ $\mathrm{cm}$ in the back muscles dorsal to the right kidney with partial compression of the pleural and retroperitoneal space (fig. 1a).

The mass surrounded the 12th rib which was fractured (black arrow in fig. 1a). The contrast enhancement of the mass was inhomogeneous with suspected necrotic areas within the tumor. There was no evidence of lymphogenic or hematogenic metastases. After discussing the case in our interdisciplinary tumor conference we decided to perform a CT-guided biopsy. The obtained core needle biopsies measured $7 \mathrm{~mm}$ in length and were whitish-reddish with a soft consistency (fig. 2a). Histological examination revealed extensive tumefactive mixed inflammatory infiltrates composed 
predominantly of diffusely distributed plasma cells and lymphoid cells in a strikingly sclerotic hyalinized background (fig. $2 \mathrm{~b}-\mathrm{d}$ ). Neutrophils and eosinophils were absent in the inflammatory infiltrates. The stromal hyalinosis was very reminiscent of amyloid deposits in some areas, but Congo Red stain (with examination under polarized light) was negative for amyloid. Small scattered follicle-like lymphoid aggregates could also be seen. A remarkable finding was the presence of isolated venous vessels with obliterated lumens as a consequence of fibrointimal dysplasia and/or mononuclear inflammatory cells within strikingly thickened intima indicating obliterative phlebitis (fig. 2b, c). However, there was no evidence of fibrinoid vascular necrosis, leukocytoclasia or granuloma. Immunohistochemistry (CD138, CD3 and CD20) confirmed a mixed inflammatory infiltrate. The lymphoid cells were of T- and B-lineage with a slight predominance of T-cells. Staining for ALK-1 was negative. Staining for IgG4 showed only rare staining cells ( $<2$ cells in 10 high power fields). The findings were then interpreted as consistent with an IPT, not otherwise specified. An FDP-PET/CT scan showed only a very mild FDP uptake. The rib fracture seemed to be an old one.

The patient was initially treated with $120 \mathrm{mg}$ prednisolone for 2 weeks which was tapered down to reach $40 \mathrm{mg}$ after 1 month. The mass began to shrink and symptoms resolved. One month after the beginning of treatment, radiological examination revealed no residual tumor tissue, indicating complete response (fig. 1b). Corticosteroid treatment was then slowly reduced to reach $5 \mathrm{mg} / \mathrm{d}$ after 6 months and than tapered off. On periodic MRT scans up to 1 year later, there was no evidence of residual tumor or tumor regrowth (fig. 1c).

\section{Discussion}

We present a case of a 26-year-old woman with an IPT at the right back mainly involving the skeletal paravertebral muscle of the back surrounding a fractured 12th rib. The cause of IPT is unclear [3]. Association between EBV and IPT of the spleen and the liver has been well established [7]. Human herpesvirus- 8 genes are suspected to play a role in pulmonary IMTs [8]. Other authors believe that autoimmunogenic processes are the underlying cause of IMT or IPT $[9,10]$. Some authors suggested that chronic infection, for example caused by long-term retention of misplaced sutures [11] or a ruptured diverticulum [12], is the underlying cause which may lead to IPTs in some of the cases.

In our case, no definite cause could be detected. It remains unclear if there is a correlation with the mild trauma experienced 6 months before. After that trauma the patient had had some pain for a few days followed by complete resolution of her symptoms for 6 months. Pain started again suddenly, which was, according to the patient's history, most likely due to a pathologic fracture of the 12th rib. Consequently, we suspect the IPT to be the cause of the pathologic fracture. However, the reverse cannot be ruled out and the relationship between the fracture of the rib and the IPT remains speculative. Furthermore, our patient did not show any signs of a predisposition for autoimmune diseases and no acute or chronic infection was known.

The pertinent differential diagnoses of this lesion include ALK-positive IMT [6], amyloid tumor/light chain disease, plasmacytoma, sclerosing low-grade malignant lymphoma, IgG4-associated systemic fibrosclerosis/pseudotumor and vasculitis. All these possibilities could be ruled out based on well-established conventional histological criteria assisted by histochemical and immunohistochemical stains. A systemic vasculitis was excluded by both clinical findings and the predominance of the extravascular inflammatory infiltrates, as well as by the absence of other more pathognomonic histological features of autoimmune vasculitis. In the literature, there are several reports in which corticosteroids where successfully used for the treatment of IPT. Most cases are described for IPT of the orbita for which usually 60 to $100 \mathrm{mg}$ prednisone per day were 
used. However, also higher doses up to 1,000 mg methylprednisolone have been administered [13]. An IPT of the lung in a 56-year-old male was successfully treated with an initial dose of $20 \mathrm{mg}$ prednisolone per day and no recurrence was observed after 10 years of follow-up [14]. In our case we used prednisolone with an initial dose of $120 \mathrm{mg} / \mathrm{d}$ for the first 2 weeks. Symptoms resolved remarkably fast with no evidence of residual tumor or tumor regrowth after 1 year.

In summary, we reported an unusual case of an IPT that resolved completely under corticosteroid treatment. It is important to recognize cases like our current case in order to avoid unnecessary radical surgery, chemotherapy, or radiation therapy, since they might be successfully treated with corticosteroids. 

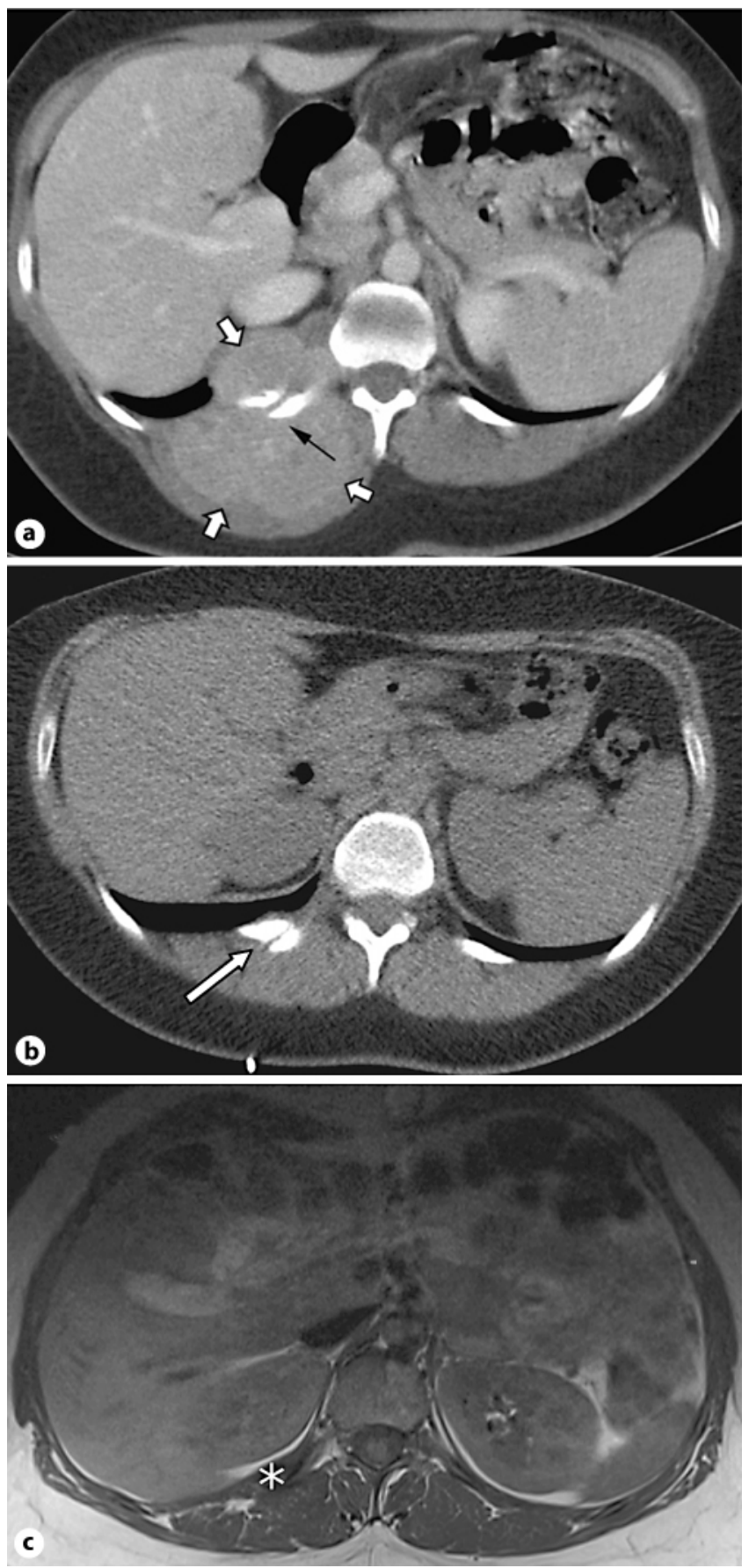

Fig. 1. a Spiral CT at the level of the upper pole of the right kidney after i.v. contrast. Enhancing mass (white arrows) with a diameter of $7.1 \times 7.3 \mathrm{~cm}$ surrounding the 12 th fractured rib (black arrow). b Spiral CT 1 month after treatment. The fractured rib is marked (white arrow). c T1-weighted MR at the level of the upper pole of the right kidney. Macroscopically complete remission of the tumor and 12 th symmetric muscles of the back. Note unchanged fracture of the rib (white asterisk). 

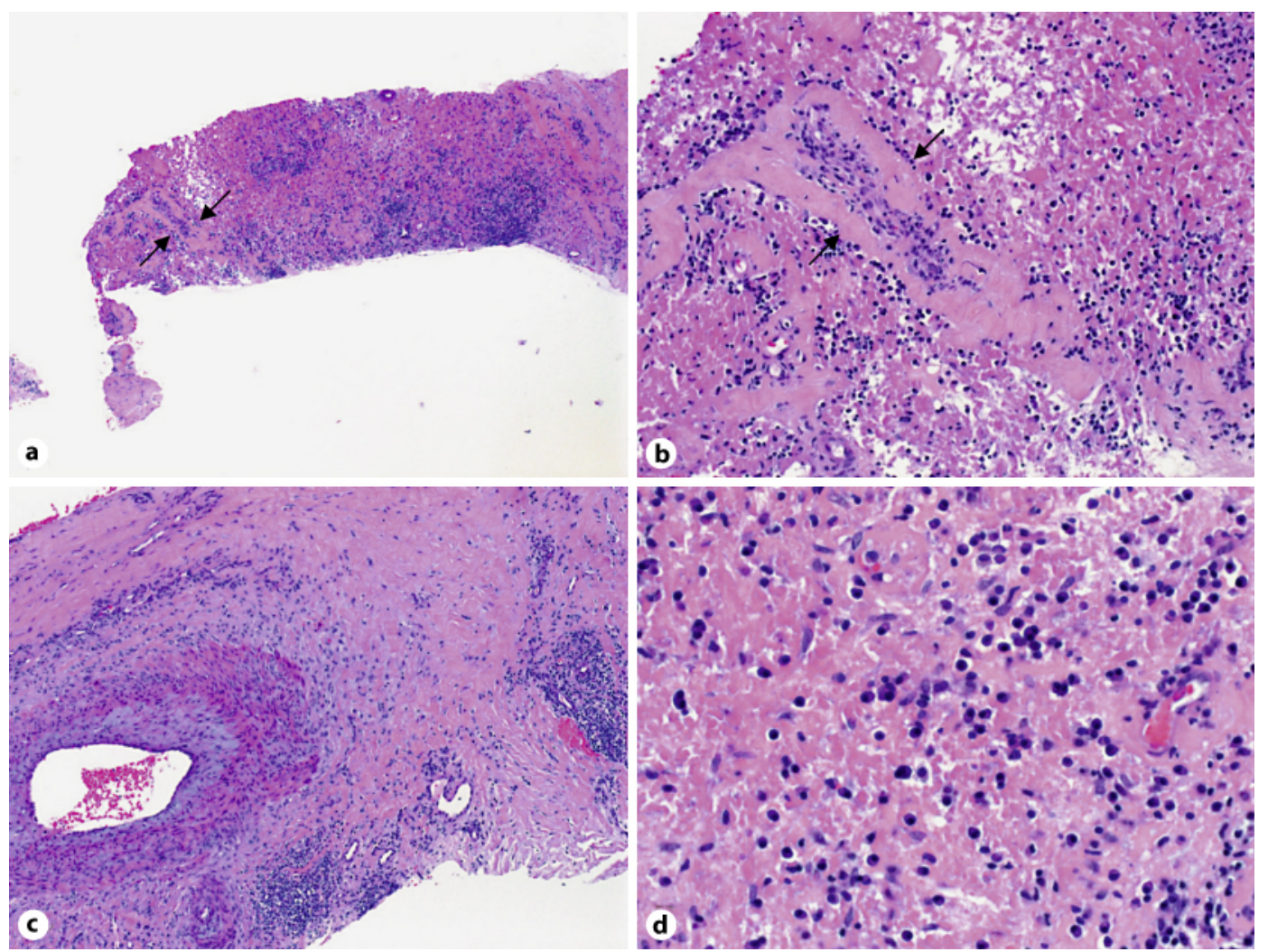

Fig. 2. a, b Tumefactive inflammatory infiltrates composed predominantly of diffusely distributed plasma cells and lymphoid cells in a strikingly sclerotic hyalinized background. A venous vessel with obliterated lumen is marked (black arrows). c Fibrointimal dysplasia was evident. d The background stromal cells showed amyloid-like hyaline changes but were negative for Congo red stain. Note prominent plasma cells.

\section{References}

1 Gleason BC, Hornick JL: Inflammatory myofibroblastic tumours: where are we now? J Clin Pathol 2008;61:428-437.

2 Cheuk W, Chan JK, Shek TW, Chang JH, Tsou MH, Yuen NW, Ng WF, Chan AC, Prat J: Inflammatory pseudotumor-like follicular dendritic cell tumor: a distinctive low-grade malignant intra-abdominal neoplasm with consistent Epstein-Barr virus association. Am J Surg Pathol 2001;25:721-731.

-3 Kutok JL, Pinkus GS, Dorfman DM, Fletcher CD: Inflammatory pseudotumor of lymph node and spleen: an entity biologically distinct from inflammatory myofibroblastic tumor. Hum Pathol 2001;32:1382-1387.

-4 Yamamoto H, Yamaguchi H, Aishima S, Oda Y, Kohashi K, Oshiro Y, Tsuneyoshi M: Inflammatory myofibroblastic tumor versus IgG4-related sclerosing disease and inflammatory pseudotumor: a comparative clinicopathologic study. Am J Surg Pathol 2009;33:1330-1340.

5 Mehta M, Jakobiec F, Fay A: Idiopathic fibroinflammatory disease of the face, eyelids, and periorbital membrane with immunoglobulin G4-positive plasma cells. Arch Pathol Lab Med 2009;133:1251-1255.

-6 Coffin CM, Hornick JL, Fletcher CD: Inflammatory myofibroblastic tumor: comparison of clinicopathologic, histologic, and immunohistochemical features including ALK expression in atypical and aggressive cases. Am J Surg Pathol 2007;31:509-520.

7 Lewis JT, Gaffney RL, Casey MB, Farrell MA, Morice WG, Macon WR: Inflammatory pseudotumor of the spleen associated with a clonal Epstein-Barr virus genome. Case report and review of the literature. Am J Clin Pathol 2003;120:56-61. 
-8 Gomez-Roman JJ, Sanchez-Velasco P, Ocejo-Vinyals G, Hernandez-Nieto E, Leyva-Cobian F, Val-Bernal JF: Human herpesvirus- 8 genes are expressed in pulmonary inflammatory myofibroblastic tumor (inflammatory pseudotumor). Am J Surg Pathol 2001;25:624-629.

-9 Mizukami H, Yajima N, Wada R, Matsumoto K, Kojima M, Kloppel G, Yagihashi S: Pancreatic malignant fibrous histiocytoma, inflammatory myofibroblastic tumor, and inflammatory pseudotumor related to autoimmune pancreatitis: characterization and differential diagnosis. Virchows Arch, 2006;448:552-560.

10 Nonaka D, Birbe R, Rosai J: So-called inflammatory myofibroblastic tumour: a proliferative lesion of fibroblastic reticulum cells? Histopathology 2005;46:604-613.

11 Jackman SV, Schulam PG, Schoenberg M: Pseudotumor of the bladder: a late complication of inguinal herniorrhaphy. Urology 1997;50:609-611.

12 Saito M, Watanabe N, Abe B, Matsui K: Inflammatory pseudotumor of the urinary bladder and sigmoid colon. Urol Int 1999;62:119-121.

13 Jacobs D, Galetta S: Diagnosis and management of orbital pseudotumor. Curr Opin Ophthalmol 2002;13:347351.

14 Ishioka S, Maeda A, Yamasaki M, Yamakido M: Inflammatory pseudotumor of the lung with pleural thickening treated with corticosteroids. Chest 2000;117:923. 